\title{
Lack of evidence of the interaction of the $A \beta$ peptide with the Wnt signaling cascade in Drosophila models of Alzheimer's disease
}

\author{
Anne-Marie Lüchtenborg and Vladimir L Katanaev*
}

\begin{abstract}
Background: Alzheimer's disease (AD) is the leading form of dementia worldwide. The A $\beta$-peptide is believed to be the major pathogenic compound of the disease. Since several years it is hypothesized that A $\beta$ impacts the Wnt signaling cascade and therefore activation of this signaling pathway is proposed to rescue the neurotoxic effect of $A \beta$.

Findings: Expression of the human A 442 in the Drosophila nervous system leads to a drastically shortened life span. We found that the action of $A \beta 42$ specifically in the glutamatergic motoneurons is responsible for the reduced survival. However, we find that the morphology of the glutamatergic larval neuromuscular junctions, which are widely used as the model for mammalian central nervous system synapses, is not affected by A 342 expression. We furthermore demonstrate that genetic activation of the Wnt signal transduction pathway in the nervous system is not able to rescue the shortened life span or a rough eye phenotype in Drosophila.

Conclusions: Our data confirm that the life span is a useful readout of A 42 induced neurotoxicity in Drosophila; the neuromuscular junction seems however not to be an appropriate model to study AD in flies. Additionally, our results challenge the hypothesis that Wnt signaling might be implicated in Aß42 toxicity and might serve as a drug target against AD.
\end{abstract}

Keywords: Alzheimer's disease, Aß peptide, Drosophila, Wnt signaling

\section{Findings}

Alzheimer's disease (AD) is a major neurodegenerative malady, affecting today more than 35 million people worldwide with the tendency to double in the prevalence every twenty years [1]. The two major hallmarks of AD are the intracellular neurofibrillary tangles consisting of the hyperphosphorylated tau protein and the extracellular plaques mainly containing the aggregated $A \beta$ peptide. According to the amyloid hypothesis, $A \beta$ peptides in their various aggregation states are the major pathogenic compounds in $\mathrm{AD}$.

Already more than a decade ago, first experiments suggested the interaction of $\mathrm{A} \beta$ with the Wnt (Wingless [Wg] in Drosophila) signaling cascade and its contribution to $A \beta$ toxicity $[2,3]$. Wnt signaling is involved in numerous developmental processes and regulates synaptic

\footnotetext{
* Correspondence: vladimir.katanaev@unil.ch
Department of Pharmacology and Toxicology, Faculty of Biology and
Medicine, University of Lausanne, Rue du Bugnon 27, Lausanne 1005,

* Correspondence: vladimir.katanaev@unil.ch
Department of Pharmacology and Toxicology, Faculty of Biology and
Medicine, University of Lausanne, Rue du Bugnon 27, Lausanne 1005,

* Correspondence: vladimir.katanaev@unil.ch
Department of Pharmacology and Toxicology, Faculty of Biology and
Medicine, University of Lausanne, Rue du Bugnon 27, Lausanne 1005, Switzerland
}

formation and stability in the adult organism [4]. In the canonical pathway, the ligand Wnt activates the receptor Frizzled (Fz) and its co-receptor LRP5/6 to induce reorganization of the $\beta$-catenin-destruction complex, a protein complex consisting of Axin, APC, glycogen synthase kinase $3 \beta$ (GSK3 $\beta$, Shaggy [Sgg] in Drosophila) and casein kinase, through the scaffolding protein Dishevelled and the trimeric Go protein $[4,5]$. Thus Wnt signaling leads to stabilization of $\beta$-catenin and its translocation to the nucleus where it induces transcription of Wnt target genes. GSK3 $\beta$ also phosphorylates tau and might be the integration point of $A \beta$ and tau induced toxicity [6].

Lithium is a well-established drug against psychiatric disorders that inhibits, amongst other targets, GSK3 $\beta$ [7]. Due to its neuroprotective effect, it has been used in small-scale trials in patients with $\mathrm{AD}$, although with contradictory results [8]. In transgenic mouse models of $\mathrm{AD}$, lithium treatment reduced behavioral impairments and the $A \beta$ load in mouse brains [9]. Likewise, the destabilization of cytosolic $\beta$-catenin and the neurotoxicity 
induced by $\mathrm{A} \beta$ in cell culture could be attenuated by $\mathrm{LiCl}$, potentially implying Wnt signaling in $A \beta$ toxicity [10]. Additionally, incubation with Wnt3a reduces the neurotoxic effect of $A \beta$ in cell culture assays - an effect mediated by Fz1 $[11,12]$. Furthermore, it has been demonstrated that the $A \beta$ peptide can bind to the receptor Fz5 [13]. Therefore, the Wnt cascade was suggested to serve as a potential drug target against $\mathrm{AD}$ [3].

Most investigations on Wnt signaling and $A \beta$ have mainly been carried out in mice and cell culture where the inhibition or activation of the signal pathway can be achieved pharmacologically. The genetic model organism Drosophila melanogaster has also been used to study the mechanisms of AD [14]. In this model, several possibilities to mimic $\mathrm{AD}$ are available; amongst others the neuronal expression of human A $\beta 42$ peptide $[15,16]$. These flies recapitulate several aspects of $\mathrm{AD}$ observed in patients: they show learning deficits, reduced locomotion, shorter life span and neurodegeneration and amyloid deposition in the brain [15].

However, the link between the Wnt signaling and $A \beta$ has not been so far investigated using the Drosophila models. We have recently provided an in-depth characterization of the Wg-Fz2-Go-Ankyrin2 signaling pathway active on the presynaptic side of Drosophila neuromuscular junctions (NMJs) [17]. NMJs are composed of synaptic boutons circular structures containing active zones for neurotransmitter release. Being a glutamatergic synapse (unlike most other synapses in Drosophila), NMJ provides an especially useful model for mammalian synapses [18]. Expression of human A $\beta 42$ in Drosophila NMJs has already been performed and reports to induce defective NMJ formation and functioning [19-21]. Thus we aimed at investigation of the details of the expected interaction of $A \beta 42$ and the Wnt signaling pathway in this system.

Pan-neuronal expression of secreted A $\beta 42$ (using the elav-Gal4 driver) has previously been shown to reduce the life span of Drosophila [15,16]. We recapitulated these findings (Figure 1A) and further showed that a similar reduction in the life span can be achieved through $A \beta 42$ expression by a motoneuron-specific driver D42-Gal4 (Figure 1B). Indeed, A $\beta 42$ caused a reduction of the median survival from 30 days (elav-Gal4 control, $\mathrm{n}=62$ ) to 10 days (elav-Gal4; UAS-A $\beta 42, \mathrm{n}=74)(\mathrm{p}<0.0001$, Logrank test) and of the maximal life span from 42 to 16 days when the pan-neuronal driver was used (Figure 1A), and from 35 days $(D 42-$ Gal4 control, $\mathrm{n}=60)$ to 12 days (D42Gal4; UAS-Aß42, $\mathrm{n}=59)(\mathrm{p}<0.0001$, Log-rank test $)$ and the maximal life span from 49 to 19 days when the motoneuron-specific driver was used (Figure 1B). This effect is dose-dependent: increasing the amount of Gal4 produced per cell by adding another motoneuron-specific driver OK371-Gal4 to D42-Gal4 to express the A 342 peptide further reduced the median life span to 10 days $(\mathrm{p}=$ 0.0028, Log-rank test) and the maximal survival to 14 days (Figure 1B). Cumulatively, these findings suggest that the major effect of $A \beta 42$ on the life span observed previously $[15,16]$ by the pan-neuronal $A \beta 42$ expression takes place in glutamatergic neurons.

We further investigated this effect of $A \beta 42$ by analyzing the morphology of the NMJs in Drosophila larvae. We expressed A 342 through elav-Gal4, D42-Gal4, and the combination of D42-Gal4 and OK371-Gal4 to increase the expression levels in the motoneurons. In contrast to previously published results where $A \beta 42$ expression induced small morphological changes in NMJs and expression of human APP and BACE led to a reduction in bouton number $[20,21]$, we could not detect an influence of $A \beta 42$ on the larval synapse. Both in terms of the overall morphology (Figure 2A) and in bouton number (Figure 2B), NMJs appear to be unaffected by $\mathrm{A} \beta 42$.

We further aimed at investigating the potential interaction of $A \beta 42$ and the Wnt signaling cascade. Given the absence of the expected phenotypes of $\mathrm{A} \beta 42$ expression
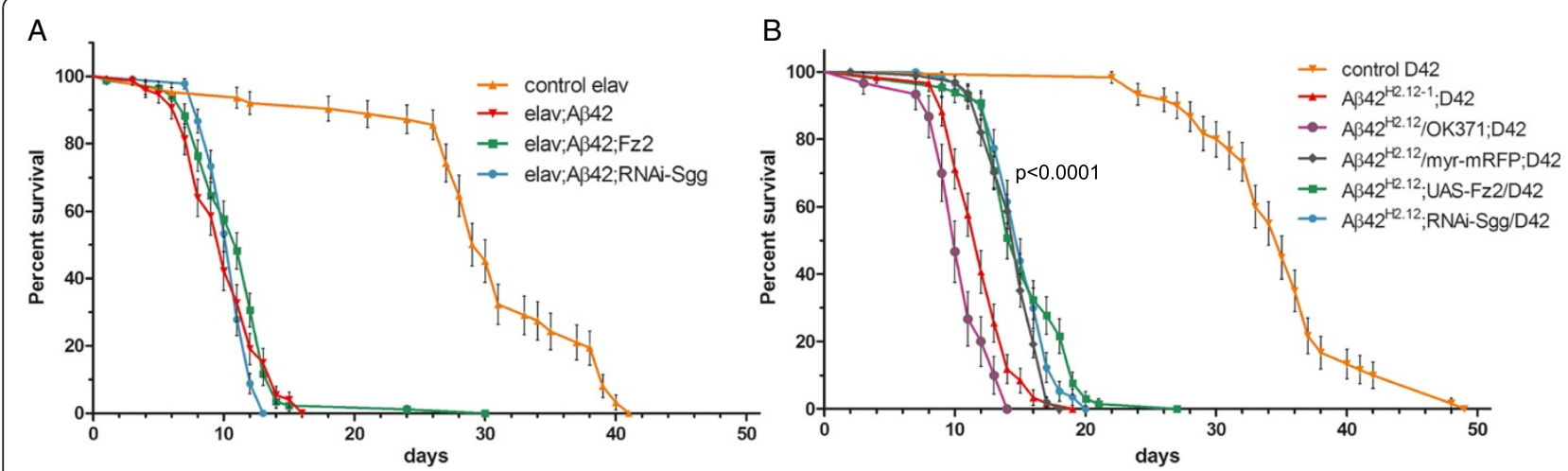

Figure 1 Expression of $\mathrm{A} \beta 42$ in the nervous system dramatically reduces the life span. (A-B) Survival curves of the indicated genotypes expressed either with the pan-neuronal driver elav-Gal4 (A) or the driver D42-Gal4 which expresses in the glutamatergic motoneurons (B). $P<0.0001$ comparing the controls elav-Ga/4 and D42-Gal4 to AB42 expression, respectively. $p=0.0028$ comparing AB42; D42-Gal4 and AB42/OK371-Gal4; D42-Gal4. Co-expression of Fz2 or RNAi-Sgg did not rescue life span. P-values are calculated with the Log-rank test. 


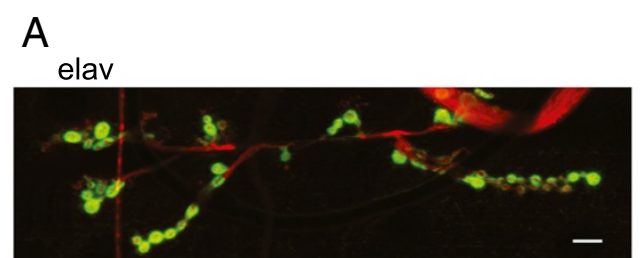

elav;A $\beta 42$

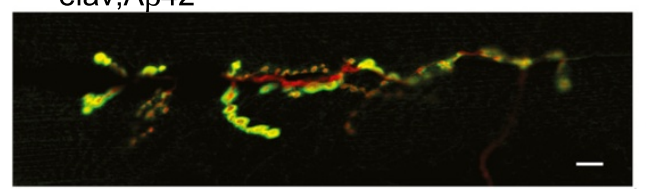

$\mathrm{D} 42 ; \mathrm{A} \beta 42$

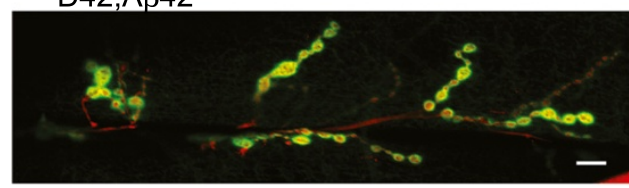

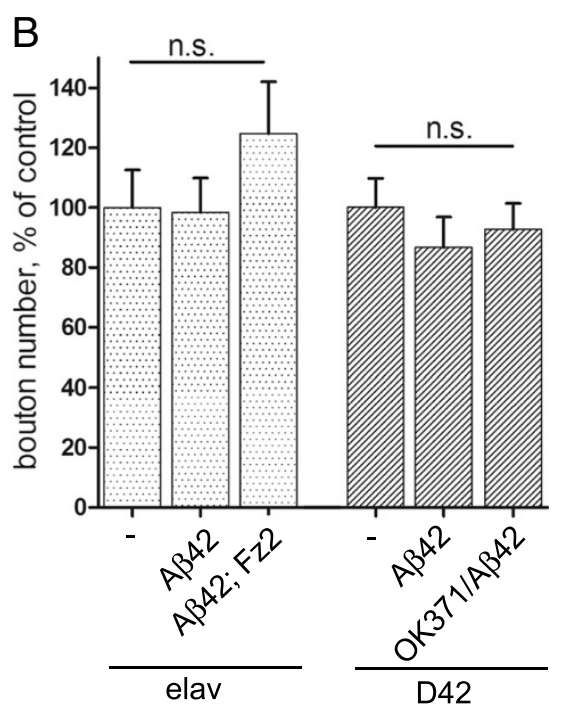

D42

Figure 2 The morphology and bouton number of NMJ are unaffected by neuronal A $\mathbf{3 4 2}$ expression. (A) Representative images of NMJS

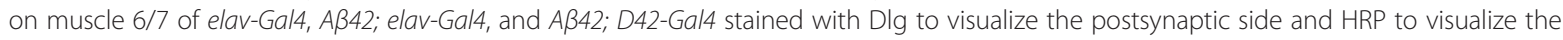
neuron. A 42 expression does not change NMJ morphology. (B) quantification of the bouton number as mean \pm sem in percent of control. n.s. means 'not significant' compared to control as calculated with student's t-test.

on NMJ morphology and bouton numbers (Figure 2), we could not use this readout to study the potential interaction between $\mathrm{A} \beta 42$ and the pathway. We thus decided to use the life span reduction as the readout. To this end, we co-expressed A 342 together with Fz2 - the main Wnt receptor in the Drosophila nervous system [17] - or together with the RNAi construct targeting Sgg to activate Wg signaling [17]. We used both the elav-Gal4 and D42Gal4 drivers. We also tried co-expression of A $\beta 42$ with

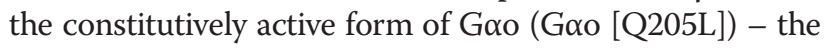
Fz2 transducer in the NMJs [17], but this was lethal with either driver, probably due to involvement of the trimeric Go protein in other neuronal activities.

In the motoneurons, co-expression of $\mathrm{Fz} 2$ or RNAiSgg together with A 342 slightly increased the survival compared to A 342 expression alone (Figure 1B). However, this was due to a titration effect since an unrelated protein (myr-mRFP) was also able to similarly rescue the life span. In pan-neuronal expression, neither Fz2 nor RNAi-Sgg could significantly increase the median survival, although the maximum life span was increased upon co-expression of Fz2 (Figure 1A). Cumulatively, these results indicate that overactivation of the Wnt signaling transduction pathways in neurons using the genetic tools available in Drosophila does not rescue the toxicity (manifested by a shortened life span) induced by secreted $A \beta 42$.

This conclusion is further corroborated using another Drosophila readout - insect's eye. A $\beta 42$ expression in the eyes using the GMR-Gal4 driver leads to a rough eye
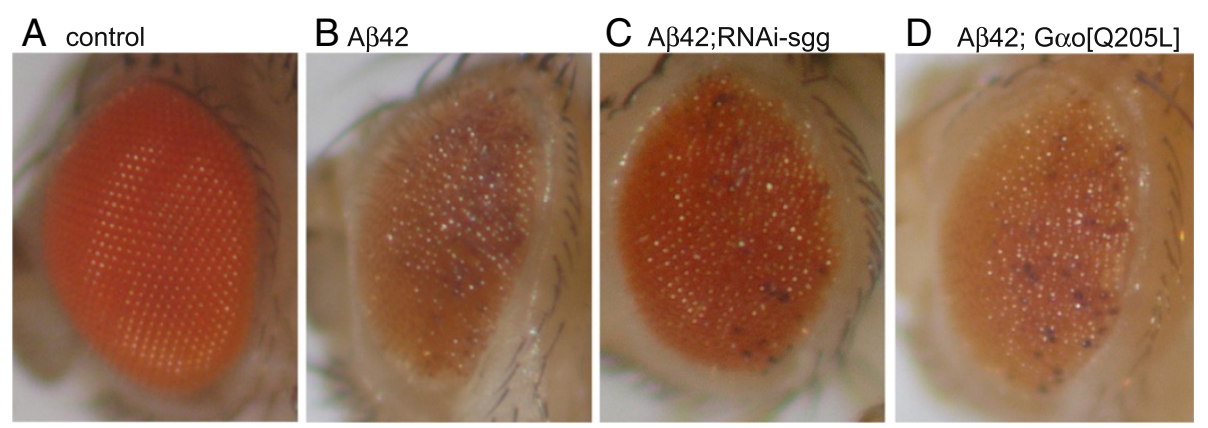

Figure 3 The rough eye phenotype induced by $\mathbf{A} 342$ is not rescued by RNAi-Sgg or Gao [Q205L]. (A) In the control eye (Aß42 without driver) ommatidia are arranged in a regular array. The other parental line, GMR-Gal4, also shows similar wild-type arrangement. (B) Expression of A 342 in the eyes with the driver GMR-Gal4 results in a rough eye phenotype. This is not rescued by co-expression of neither RNAi-Sgg (C) nor Gao [Q205L] (D). 
phenotype (Figure 3, [16]). We co-expressed RNAi-Sgg and Goo [Q205L] with $A \beta 42$ and could not observe a rescue of the eye roughness (Figure 3). This confirms in a different setting that genetic activation of the Wnt signaling cascade does not rescue A $\beta 42$ induced toxicity in Drosophila.

However, it has been previously reported that expression of the dominant-negative form of Sgg, SggS9E, rescues the shortened life span of Drosophila that express the arctic Aß42 peptide [22]. The arctic peptide was shown to decrease Ser9 phosphorylation of Sgg and thereby upregulate its activity [22]. In contrast another study shows no change in phosphorylation of Sgg when wild-type $A \beta 42$ is expressed [20]. The arctic variant accumulates intracellularly in mice $[23,24]$; therefore it is likely that the arctic variant of $A \beta 42$ exerts a different pathogenic mechanism than the wild-type peptide. Our data suggest that secreted wild-type $A \beta 42$ acts primarily on glutamatergic neurons in Drosophila, but induces the toxicity independently from Wnt signaling.

Taken together, our results demonstrate that A $\beta 42$ expression in glutamatergic neurons is responsible for the dramatic shortened life span manifested in Drosophila models of $\mathrm{AD}$. However, the glutamatergic NMJs seem not to be appropriate to study the A $\beta 42$-induced changes on a single cell level. In addition, our genetic interaction analysis challenges the widely accepted idea that $A \beta 42$ inhibits Wnt signaling and that Wnt pathway overactivation might reduce the A $\beta 42$ toxicity. This concept is based on numerous studies, mainly relying on the usage of $\mathrm{LiCl}$ and its effects on the $\mathrm{AD}$ phenotypes. We suggest that caution is taken when interpreting these data, as $\mathrm{LiCl}$ is not a specific inhibitor of GSK3 $\beta / \mathrm{Sgg}$, and further since this kinase has many other functions outside the Wnt signaling pathway [7].

\section{Methods}

\section{Life span, eye analysis and Drosophila stocks}

For the life span test, flies were crossed at $25^{\circ} \mathrm{C}$ and 5 male and 5 female newly hatched flies were pooled and transferred to $28.5^{\circ} \mathrm{C}$. Flies were transferred to fresh food every other day and time-to-death was recorded for individual flies. 59 to 90 flies were analyzed for each genotype. Analysis of survival was performed with GraphPad Prism 5, p-values were calculated with the Log-rank test.

Eye phenotypes were analyzed after crossing to GMRGal4 at $25^{\circ} \mathrm{C}$.

The following stock were used: elav-Gal4, D42-Gal4, GMR-Gal4 (all from Bloomington stock center), UASRNAi-Sgg (VCRC \#7005), UAS-AB42 [16]. The lines

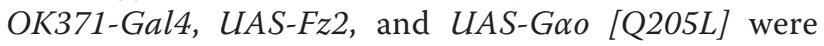
used as described [17].

\section{Immunohistochemistry}

For the analysis of the neuromuscular junctions, crosses were set up at $28.5^{\circ} \mathrm{C}$ and wandering third instar larvae were dissected and stained as previously described [17]. Primary antibodies were: Cy3-coupled goat anti-HRP (123-165-021, Jackson ImmunoResearch) at 1:200 and mouse anti-Dlg (4 F3, Developmental Studies Hybridoma Bank) at 1:100. Boutons were identified based on presynaptic HRP and postsynaptic Dlg staining. Statistical analysis was performed with GraphPad Prism 5. Data are present as mean \pm sem.

\section{Competing interests}

The authors declare that they have no competing interests.

\section{Authors' contributions}

AML designed, performed and interpreted the data and wrote the manuscript; VLK designed and interpreted the data and wrote the manuscript. Both authors read and approved the final manusrcipt.

\section{Acknowledgements}

We thank Gonzalo Solis for critically reading the manuscript. The work was funded by a grant of Synapsis Foundation to VLK.

Received: 27 August 2014 Accepted: 28 October 2014

Published online: 12 November 2014

\section{References}

1. Prince M, Bryce R, Albanese E, Wimo A, Ribeiro W, Ferri CP: The global prevalence of dementia: a systematic review and metaanalysis. Alzheimers Dement 2013, 9:63-75.e62.

2. Inestrosa NC, Alvarez A, Godoy J, Reyes A, De Ferrari GV: Acetylcholinesterase-amyloid-beta-peptide interaction and Wnt signaling involvement in Abeta neurotoxicity. Acta Neurol Scand Suppl 2000, 176:53-59.

3. Inestrosa NC, Varela-Nallar L: Wnt signaling in the nervous system and in Alzheimer's disease. J Mol Cell Biol 2014, 6:64-74.

4. Blagodatski A, Poteryaev D, Katanaev V: Targeting the Wnt pathways for therapies. Mol Cell Ther 2014, 2:28.

5. Egger-Adam D, Katanaev VL: The trimeric $G$ protein go inflicts a double impact on axin in the Wnt/frizzled signaling pathway. Dev Dyn 2010, 239:168-183.

6. Qu ZS, Li L, Sun XJ, Zhao YW, Zhang J, Geng Z, Fu JL, Ren QG: Glycogen synthase kinase- 3 regulates production of amyloid- beta peptides and Tau phosphorylation in diabetic Rat brain. Sci World J 2014, 2014:878123.

7. O'Brien WT, Klein PS: Validating GSK3 as an in vivo target of lithium action. Biochem Soc Trans 2009, 37:1133-1138.

8. Forlenza OV, de Paula VJ, Machado-Vieira R, Diniz BS, Gattaz WF: Does lithium prevent Alzheimer's disease? Drugs Aging 2012, 29:335-342.

9. Toledo EM, Inestrosa NC: Activation of Wnt signaling by lithium and rosiglitazone reduced spatial memory impairment and neurodegeneration in brains of an APPswe/PSEN1DeltaE9 mouse model of Alzheimer's disease. Mol Psychiatry 2010, 15:272-285. 228.

10. De Ferrari GV, Chacon MA, Barria MI, Garrido JL, Godoy JA, Olivares G, Reyes AE, Alvarez A, Bronfman M, Inestrosa NC: Activation of Wnt signaling rescues neurodegeneration and behavioral impairments induced by beta-amyloid fibrils. Mol Psychiatry 2003, 8:195-208.

11. Alvarez AR, Godoy JA, Mullendorff K, Olivares GH, Bronfman M, Inestrosa NC: Wnt-3a overcomes beta-amyloid toxicity in rat hippocampal neurons. Exp Cell Res 2004, 297:186-196.

12. Chacon MA, Varela-Nallar L, Inestrosa NC: Frizzled-1 is involved in the neuroprotective effect of Wnt3a against Abeta oligomers. J Cell Physiol 2008, 217:215-227.

13. Magdesian $M H$, Carvalho MM, Mendes FA, Saraiva LM, Juliano MA, Juliano L, Garcia-Abreu J, Ferreira ST: Amyloid-beta binds to the extracellular cysteine-rich domain of Frizzled and inhibits Wnt/beta-catenin signaling. J Biol Chem 2008, 283:9359-9368.

14. Prussing K, Voigt A, Schulz JB: Drosophila melanogaster as a model organism for Alzheimer's disease. Mol Neurodegener 2013, 8:35.

15. lijima K, Liu HP, Chiang AS, Hearn SA, Konsolaki M, Zhong Y: Dissecting the pathological effects of human Abeta40 and Abeta42 in drosophila: a 
potential model for Alzheimer's disease. Proc Natl Acad Sci U S A 2004, 101:6623-6628.

16. Sanokawa-Akakura R, Cao W, Allan K, Patel K, Ganesh A, Heiman G, Burke R, Kemp FW, Bogden JD, Camakaris J, Raymond BB, Konsolaki M: Control of Alzheimer's amyloid beta toxicity by the high molecular weight immunophilin FKBP52 and copper homeostasis in Drosophila. PLoS One 2010, 5:e8626.

17. Luchtenborg AM, Solis GP, Egger-Adam D, Koval A, Lin C, Blanchard MG, Kellenberger S, Katanaev VL: Heterotrimeric Go protein links Wnt-Frizzled signaling with ankyrins to regulate the neuronal microtubule cytoskeleton. Development 2014, 141:3399-3409.

18. Collins CA, DiAntonio A: Synaptic development: insights from drosophila. Curr Opin Neurobiol 2007, 17:35-42.

19. Chiang HC, lijima K, Hakker I, Zhong Y: Distinctive roles of different beta-amyloid 42 aggregates in modulation of synaptic functions. FASEB $J$ 2009, 23:1969-1977.

20. Folwell J, Cowan CM, Ubhi KK, Shiabh H, Newman TA, Shepherd D, Mudher A: Abeta exacerbates the neuronal dysfunction caused by human tau expression in a Drosophila model of Alzheimer's disease. Exp Neurol 2010, 223:401-409.

21. Mhatre SD, Satyasi V, Killen M, Paddock BE, Moir RD, Saunders AJ, Marenda DR: Synaptic abnormalities in a Drosophila model of Alzheimer's disease. Dis Model Mech 2014, 7:373-385.

22. Sofola O, Kerr F, Rogers I, Killick R, Augustin H, Gandy C, Allen MJ, Hardy J, Lovestone S, Partridge L: Inhibition of GSK-3 ameliorates Abeta pathology in an adult-onset Drosophila model of Alzheimer's disease. PLoS Genet 2010, 6:e1001087.

23. Lord A, Kalimo H, Eckman C, Zhang XQ, Lannfelt L, Nilsson LN: The arctic Alzheimer mutation facilitates early intraneuronal Abeta aggregation and senile plaque formation in transgenic mice. Neurobiol Aging 2006, 27:67-77.

24. Sahlin C, Lord A, Magnusson K, Englund H, Almeida CG, Greengard P, Nyberg F, Gouras GK, Lannfelt L, Nilsson LN: The arctic Alzheimer mutation favors intracellular amyloid-beta production by making amyloid precursor protein less available to alpha-secretase. J Neurochem 2007, 101:854-862

doi:10.1186/s13041-014-0081-y

Cite this article as: Lüchtenborg and Katanaev: Lack of evidence of the interaction of the $A \beta$ peptide with the Wnt signaling cascade in Drosophila models of Alzheimer's disease. Molecular Brain 2014 7:81.

\section{Submit your next manuscript to BioMed Central and take full advantage of:}

- Convenient online submission

- Thorough peer review

- No space constraints or color figure charges

- Immediate publication on acceptance

- Inclusion in PubMed, CAS, Scopus and Google Scholar

- Research which is freely available for redistribution

Submit your manuscript at www.biomedcentral.com/submit
(O) Biomed Central 